\title{
New empirical methods for correction of meteorological effects on cosmic ray muons
}

\section{Savić,,$^{a * *}$ A. Dragić, ${ }^{a}$ D. Maletić, ${ }^{a}$ N. Veselinović, ${ }^{a}$ D. Joković, ${ }^{a}$ R. Banjanac, ${ }^{a}$ V. Udovičić $^{a}$ and D. Knežević ${ }^{a}$}

${ }^{a}$ Institute of Physics Belgrade,

Pregrevica 118, 11080 Belgrade, Serbia

E-mail: msavic@ipb.ac.rs

Flux of muon component of secondary cosmic rays is affected by varying conditions in the atmosphere. Dominant effects are barometric and temperature effect, which reflect variations of atmospheric pressure and atmospheric temperature respectively. Precise modelling and correction for these meteorological effects significantly increases sensitivity of Earth-based muon detectors to variations of primary cosmic ray flux. We are presenting two recently developed empirical methods for correction of meteorological effects on cosmic ray muons. First method is based on principal component analysis, while second employs multivariate analysis using machine learning techniques. Both methods are applied for correction of barometric and temperature effects, but can easily be generalised to take more atmospheric parameters into account. We apply these corrections to muon count rates measured by Belgrade cosmic ray station and study their effect on sensitivity of detection of periodic and aperiodic flux variations of primary cosmic rays. Comparison with the most widely used method for correction of meteorological effects - integral method, as well as with neutron monitor data, demonstrates very high effectiveness of presented methods.

$37^{\text {th }}$ International Cosmic Ray Conference (ICRC 2021)

July 12th - 23rd, 2021

Online - Berlin, Germany

\footnotetext{
*Presenter
} 


\section{Introduction}

Cosmic ray muons (hard component of secondary cosmic rays) are affected by variations of atmospheric parameters as they propagate toward Earth. There are a number of meteorological effects that affect cosmic ray muon flux, most prominent being the barometric (pressure) effect and the temperature effect, which depend on atmospheric pressure and atmospheric temperature respectively. Apart from fundamental, precise modelling of these effects also has practical importance, as it allows for correction that significantly increases the sensitivity of ground based muon monitors to variations of primary cosmic rays.

A number of methods for correction of barometric and temperature effect have been developed over the years. Some (i.e. method of effective level of generation [1]) are empirical in nature, while others (most notably integral method) rely on the theory of meteorological effects, developed by Dorman [2] among others. All these methods are at least in some part approximative, but for all intents and purposes we have decided to use the integral method as a reference in our analysis, as it gives the most complete treatment of the problem.

The idea behind the work presented here is to try and develop a new, easy to use empirical method, less approximative in nature, compare it to the reference integral method, and investigate whether a more precise model of meteorological effects can be constructed, and possibly some additional information extracted. In order to most completely treat the meteorological effects, both atmospheric pressure and full atmospheric temperature profile need to be taken into account. For analysis that involves that many potentially highly correlated input variables, we have decided to employ modern techniques used for decorrelation and dimensionality reduction, and introduce two new methods for modelling and correction of meteorological effects - PCA method based on principal component analysis (PCA), and MVA method based on multivariate analysis (MVA) via use of machine learning. Though these two are somewhat similar in nature, a more "hands on" approach of the PCA method can offer a somewhat different insight than the more "blackbox" machine learning approach.

\section{Data}

\subsection{CR data}

Muon count rates used in this analysis were measured in the Ground Level Laboratory (GLL) of the Low Background Laboratory for Nuclear Physics, at the Institute of Physics Belgrade [3]. More detailed description of the laboratory and current detector system can be found in some of our previous work [4]. Muon count rates can have arbitrary time resolution but five-minute and hour sums were used in the analysis. For quality and consistency of data reasons, and to remove potential biases due to annual variation, data for a period of one year (from 01.06.2010 to 31.05.2011) were selected.

\subsection{Meteo data}

This analysis requires information about both atmospheric pressure and vertical atmospheric temperature profile. Data about atmospheric pressure is readily available from the Republic Hydrometeorological Servis of Serbia. As for the vertical temperature profile data, temperatures for 24 

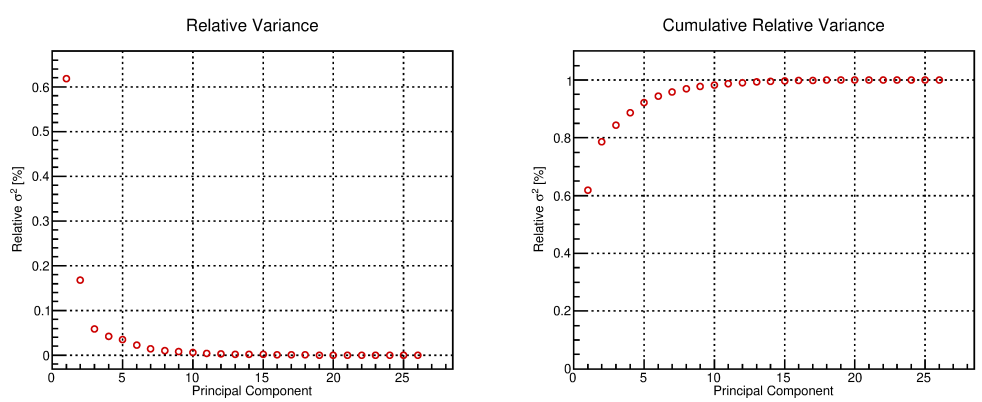

Figure 1: Relative variance (left) and cumulative relative variance (right) for all 26 principal components.

isobaric levels modelled by the Global Forecast System (GFS) [5] were used, starting from the top layer of the atmosphere $(10 \mathrm{mb})$, to the level just above ground level $(975 \mathrm{mb})$. For the above ground layer, locally measured temperature was used as the model was performing poorly there. More details about the preparation of meteorological data is available elsewhere [7].

\section{Methodology}

\subsection{PCA method}

Principal component analysis is a well established technique for dimensionality reduction of complex problems that involve large number of correlated variables, and as such very well suited for application to our problem. Using principal component decomposition we have transformed the initial set of correlated meteorological variables (locally measured atmospheric pressure, 24 modelled temperatures, and locally measured ground temperature) to a set of 26 uncorrelated principal components.

Using a series of tests typically used in such analysis (cumulative percentage rule, modified Kaisser's rule, mean eigenvalue rule, ...), we have determined that the first six components (responsible for close to $95 \%$ of total variance, as seen on Figure 1) are significant. Composition of the these components is shown on Figure 2, where variables on the $\mathrm{x}$-axis are atmospheric pressure followed by atmospheric temperatures, starting from the top layer of the atmosphere.

Correlative analysis of muon count rate and significant principal components showed practically no correlation between measured muon count rate and the second principal component, further reducing the set of principal components to five. This is an interesting results as this component, mainly composed of lower stratosphere and upper troposphere temperatures, is responsible for close to $17 \%$ of total variation of meteorological variables.

Finally, we have determined the muon count rate corrected for meteorological effects according to formula:

$$
N_{\mu}^{(\text {corr })}=N_{\mu}-\left\langle N_{\mu}\right\rangle \sum_{i} k_{i} P C_{i}, \quad i=1,3,4,5,6
$$

where $N_{\mu}^{(c o r r)}$ is corrected, $N_{\mu}$ measured and $\left\langle N_{\mu}\right\rangle$ mean muon count rate, while $k_{i}$, that correspond to principal components $P C_{i}$, are coefficients determined by linear regression, as shown on Figure 3. Full analysis and results are presented in more detail in our other work [8]. 

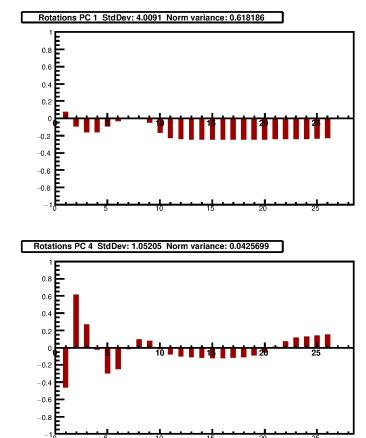
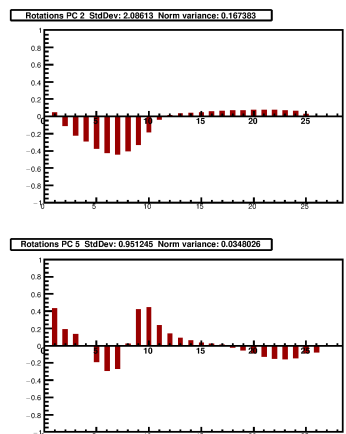
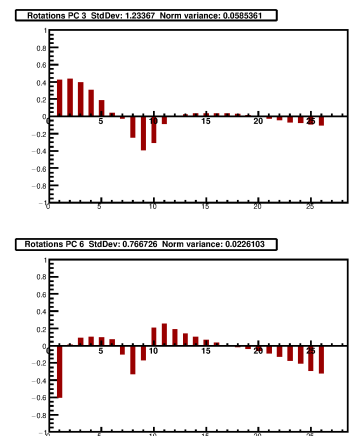

Figure 2: Composition for six most significant principal components. Meteorological variables are on the $\mathrm{x}$-axis, first one being atmospheric pressure, followed by atmospheric temperatures (starting with the top layer of the atmosphere and ending with the ground level).
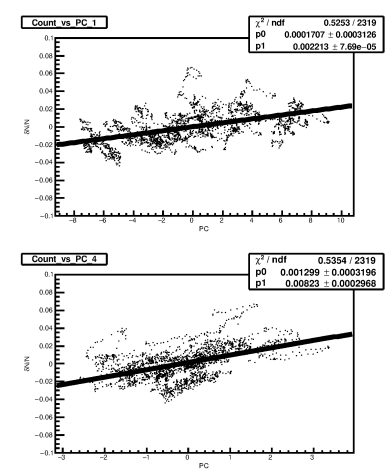
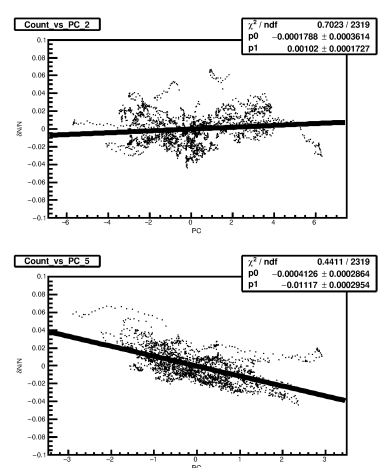
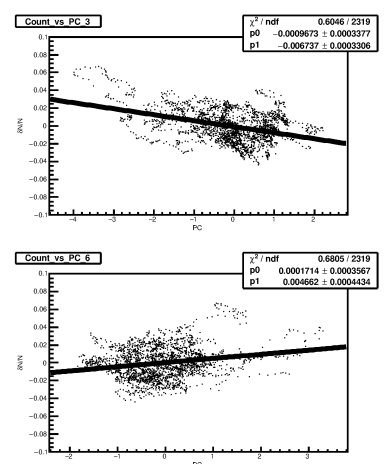

Figure 3: Muon count rate dependence on principal components for six most significant components, distributions fitted with linear function.

\subsection{MVA method}

Multivariate analysis utilising machine learning techniques can be a powerful tool for modelling of highly correlated systems. We have tested a number of algorithms implemented in Toolkit for Multivariate Data Analysis (TMVA), which has been successfully used for classification and regression problems in particle physics. For us, regression application is of greater interest, as the idea is to train and test multivariate algorithms on a subset of data (for geomagnetically quiet days), where most of the variation can be attributed to atmospheric effects, using meteorological variables as input and muon count rate as the target value. Trained algorithms can be then used on a full data set to predict the muon count rate (which would ideally depend only on meteorological parameters), and corrected muon count rate can be calculated using the formula:

$$
N_{\mu}^{(\text {corr })}=\Delta N_{\mu}+\left\langle N_{\mu}\right\rangle, \quad \Delta N_{\mu}=N_{\mu}^{(\text {mod })}-N_{\mu},
$$

where $N_{\mu}^{(\text {corr })}$ is corrected, $N_{\mu}$ measured, $N_{\mu}^{(\text {mod })}$ modelled, and $\left\langle N_{\mu}\right\rangle$ is mean muon count rate.

Minimal average quadratic deviation of modelled from measured value was the only criterion used for optimisation of algorithm parameters in the training phase, so a series of tests have been 

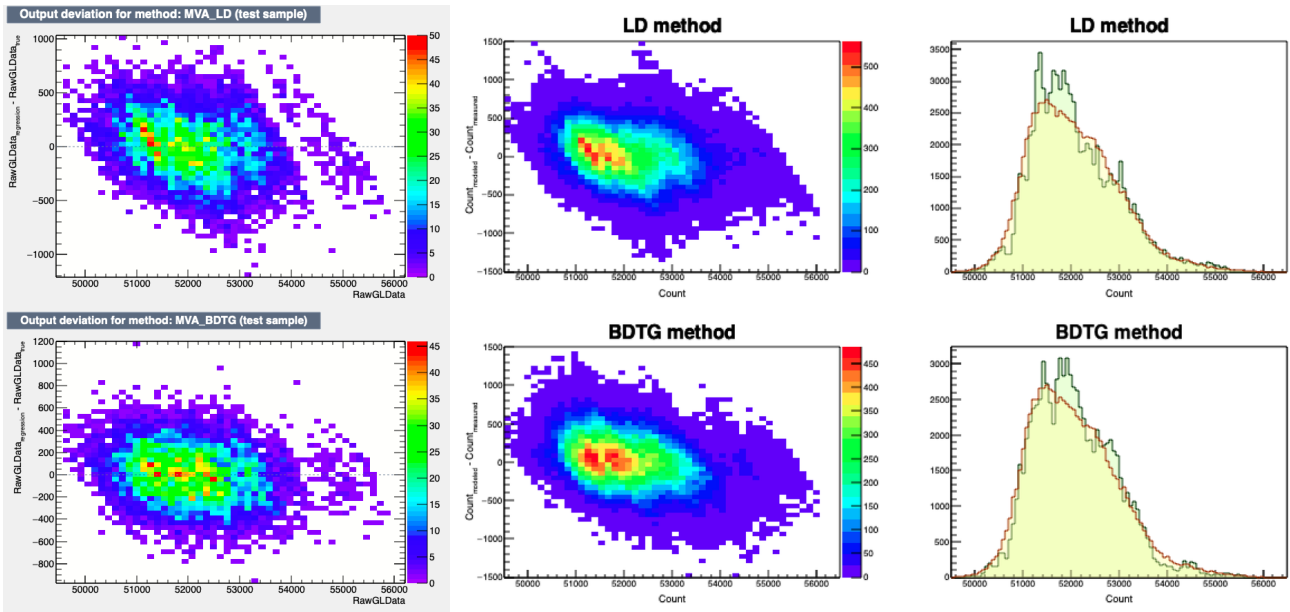

Figure 4: Modelled count rate and its deviation from measured count rate as a function of measured count rate for LD (top) and BDTG (bottom) algorithms. Deviation distributions for test data set are on left, for the full data set are in the middle, while distributions of modelled count rate (compared with the measured one) are on the right.
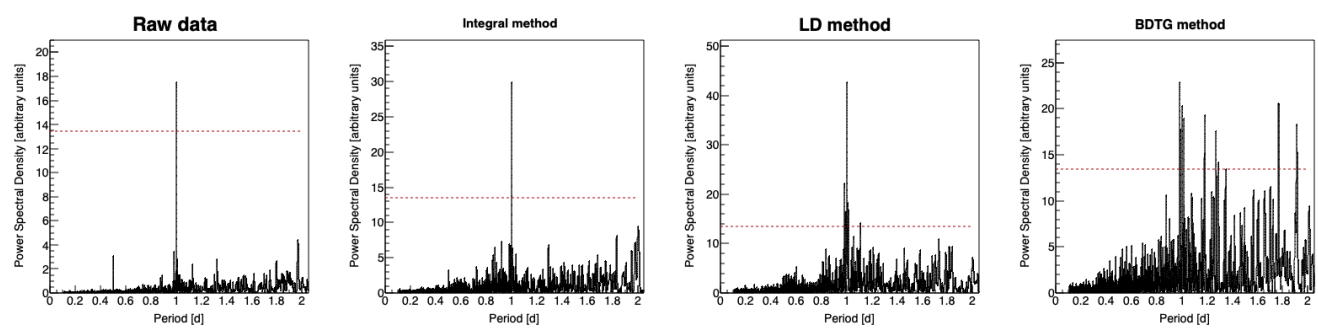

Figure 5: Power spectra for periods in the interval [0, 2] days, for measured data (far left), and data corrected using integral (central left), LD (central right) and BDTG (far right) methods.

devised in order to investigate the consistency of application of trained algorithms and minimise the possibility of artificial features being introduced.

Some of the tests included comparison of distributions of residual deviation of modelled from measured data for the test and full data set, or looking for anomalous features in distributions of modelled count in comparison with measured count distribution (both types of distributions for selected algorithms shown in Figure 4.

Based on these tests, the best performing algorithm proved to be LD (Linear Discriminant method), which is closely related to PCA approach. The second best potential candidate was BDTG (Gradient Boosted Decision Tree method), but there are probably some limits to its applicability, as indicated by spectral analysis (Figure 5). From the remaining tested methods, algorithms based on probability density techniques performed more poorly, which was not that surprising as the problem analysed here involves highly linear dependencies, but poor performance of methods based on neural networks was not expected, and possibly some improvement can be made there. 

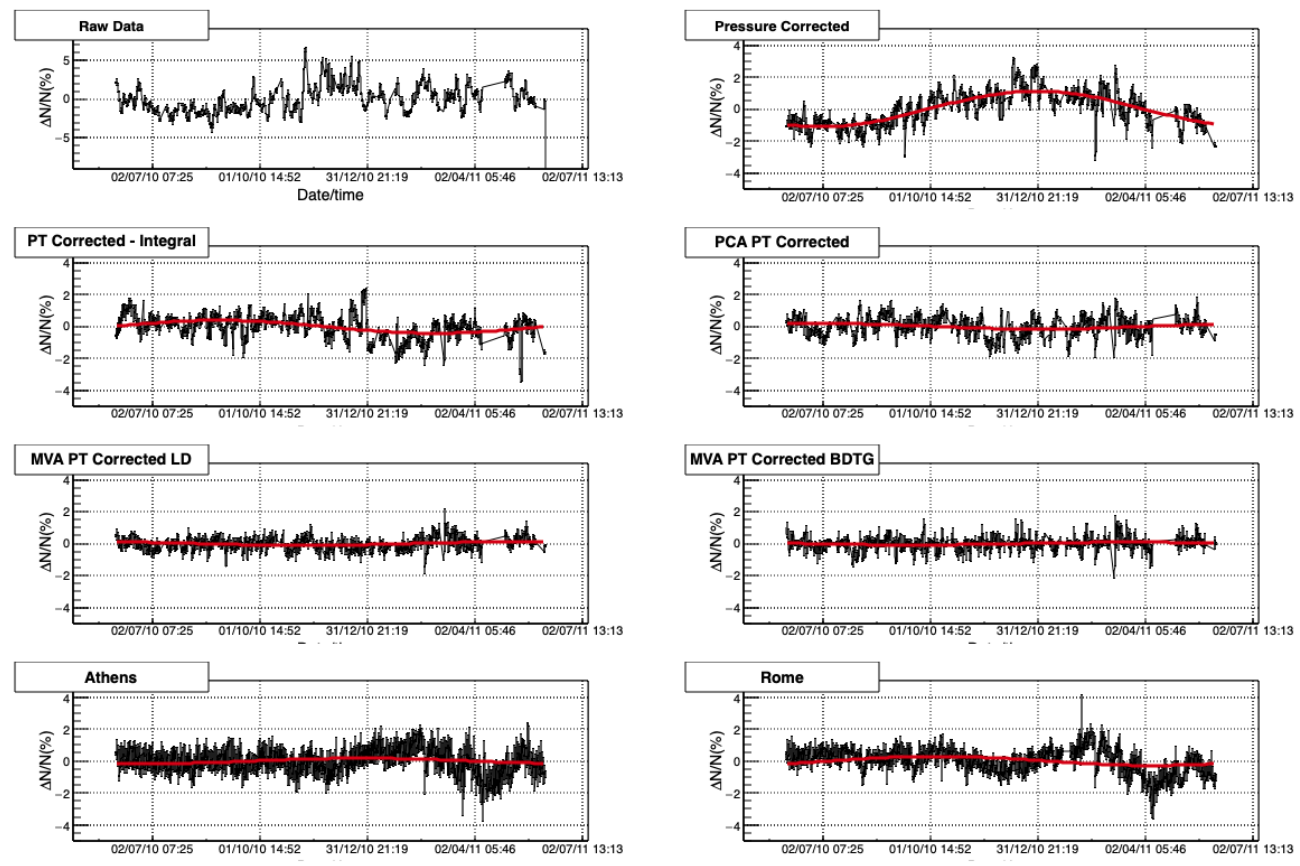

Figure 6: Muon count rate time series and reference neutron monitor data for the period of one year (01.06.2010-31.05.2011), fitted with sine function with a period of one year.

\section{Results}

\subsection{Effect of corrections on periodic CR variations}

One way to assess the performance of different methods for correction of meteorological effects could be to compare the efficiency with which they remove the annual variation due to temperature variation. In order to determine this variation, we have fitted pressure corrected data with a sine function, with a period of one year. Amplitude determined from such fit is then used as an estimate of magnitude of the annual variation. The same procedure was used to determine the residual annual variation after the correction via use of different methods (Figure 6). As neutron monitor count rates are usually considered to negligibly depend on atmospheric temperature (at least in the first approximation), we can treat their time series the same way in order to estimate the expected annual variation magnitude.

Table 1 shows amplitudes for the annual variation calculated based on plots in Figure 6, as well as reduction in annual variation relative to pressure corrected data. As can be seen, values for PCA and LD methods are closer to the estimates based on the neutron monitor data than the integral method value, while for BDTG method the value is somewhat smaller.

\subsection{Effect of corrections on aperiodic CR variations}

To study the effect of corrections on aperiodic variations we have selected the most intense Forbush decrease event in the one year period used for the analysis. For the event that occurred on 18.02.2011, we determined the amplitude of decrease for data corrected via different methods and reference neutron monitors, using procedure suggested by Barbashina et al. [9] (as shown on 


\begin{tabular}{cccccccc}
\hline $\begin{array}{c}\text { Method/ } \\
\text { Neutron monitor }\end{array}$ & P corr. & Integral & PCA & LD & BDTG & Athens & Rome \\
\hline $\begin{array}{c}\text { Annual } \\
\text { amplitude [\%] }\end{array}$ & $1.11(9)$ & $0.40(3)$ & $0.18(5)$ & $0.11(3)$ & $0.086(9)$ & $0.17(5)$ & $0.29(1)$ \\
\hline $\begin{array}{c}\text { Relative reduction } \\
{[\% \text { of P corrected }]}\end{array}$ & - & $64(10)$ & $84(28)$ & $90(30)$ & $92(30)$ & - & - \\
\hline
\end{tabular}

Table 1: Amplitude and reduction of the amplitude of annual variation relative to pressure corrected data (P corr.) for pressure and temperature corrected data (using integral and selected multivariate methods). Athens and Rome neutron monitor data also included for reference
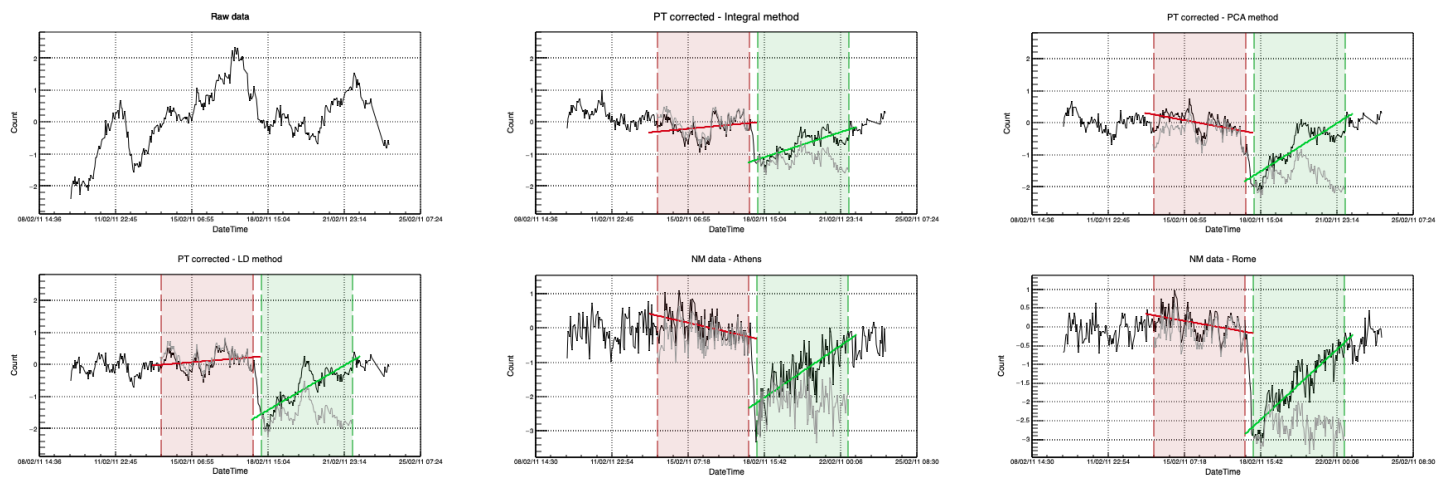

Figure 7: Muon count rate time series and reference neutron monitor data for the period around the Forbush decrease event of 18.02.2011. Highlighted intervals are used for detrending and calculation of decrease amplitude.

\begin{tabular}{ccccccc}
\hline $\begin{array}{c}\text { Method/ } \\
\text { Neutron monitor }\end{array}$ & Integral & PCA & LD & BDTG & Athens & Rome \\
\hline $\begin{array}{c}\text { FD } \\
\text { amplitude [\%] }\end{array}$ & $1.38(14)$ & $1.52(21)$ & $1.96(18)$ & $1.10(13)$ & $1.97(15)$ & $2.68(15)$ \\
\hline $\begin{array}{c}\text { Relative FD } \\
\text { amplitude }\end{array}$ & $4.31(44)$ & $4.90(66)$ & $7.09(65)$ & $4.78(56)$ & $5.30(40)$ & $8.65(48)$ \\
\hline
\end{tabular}

Table 2: Amplitudes and relative amplitudes for the Forbush decrease event of 18.02.2011 for pressure and temperature corrected muon data and reference neutron monitors

Figure 7). Additionally, as a measure of sensitivity to such events, we have introduced amplitude calculated relative to standard deviation of count rates leading up to the event.

Values for thusly calculated amplitudes and relative amplitudes are shown in Table 2. LD algorithm has values comparable to neutron monitor values, but that is at least in part due to somewhat larger calculated amplitude. This is most likely a feature pertaining to the specific event, as preliminary results for other events outside the interval used in this work show values closer to expected. 


\section{Conclusions}

Two new methods for correction of meteorological effects on cosmic ray muons are introduced. Both are fully empirical, require knowledge about the atmospheric pressure and atmospheric temperature profile and can be applied to any muon monitor. The effect on reduction of the annual variation of CR data, as well as the effect on sensitivity of FD event detection was compared to the integral method and reference neutron monitor data. Their effectiveness was comparable or possibly better than for the integral method, allowing for the possibility that a part of meteorological effects is not taken into account by theory.

\section{References}

[1] A Duperier, "The Meson Intensity at the Surface of the Earth and the Temperature at the Production Level", in Proceedings of the Physical Society. Section A, volume 62, number 11, pages 684, year 1949, doi: 10.1088/0370-1298/62/11/302

[2] Dorman, L. I., "On the temperature effect of the hard component of cosmic rays", in Reports of Academy of Sciences of USSR (DAN SSSR), volume 95, issue 1, pages 49 - 52, year 1954b

[3] Low Background Laboratory for Nuclear Physics, url: http://www.cosmic.ipb.ac.rs/

[4] Dragic Aleksandar, Udovicic Vladimir, Banjanac Radomir, Jokovic Dejan, Maletic Dimitrije, Veselinovic Nikola, Savic Mihailo, Puzovic Jovan, Anicin Ivan, "The New Set-Up in the Belgrade Low-Level and Cosmic-Ray Laboratory", in NUCLEAR TECHNOLOGY AND RADIATION PROTECTION, volume 26, number 3, pages 181-192, year 2011, doi: 10.2298/NTRP1103181D

[5] GFS, url: https://www.ncdc.noaa.gov/data-access/model-data/model-datasets/global-forcastsystem-gfs

[6] Berkova M., Belov A., Eroshenko E., Yanke Victor, "Temperature effect of muon component and practical questions of how to take into account in real time", in Astrophysics and Space Sciences Transactions, year 2012, pages 41-44, volume 8, doi: 10.5194/astra-8-41-2012

[7] Savic Mihailo, Dragic Aleksandar, Veselinovic Nikola, Udovicic Vladimir, Banjanac Radomir, Jokovic Dejan, Maletic Dimitrije, "Effect of pressure and temperature corrections on muon flux variability at ground level and underground", in "25th European Cosmic Ray Symposium", Torino, Italy, eprint: 1701.00164, year 2016

[8] Savic Mihailo, Dragic Aleksandar, Maletic Dimitrije, Veselinovic Nikola, Banjanac Radomir, Jokovic Dejan, Udovicic Vladimir, "A novel method for atmospheric correction of cosmic-ray data based on principal component analysis", in ASTROPARTICLE PHYSICS, volume 109, pages 1-11, year 2019, doi: 10.1016/j.astropartphys.2019.01.006

[9] Barbashina N., Dmitrieva Anna, Kompaniets K., Petrukhin A., Timashkov D., Shutenko V., Yakovleva Elena, Yashin, Igor, "Specific features of studying Forbush decreases in the muon flux", in Bulletin of The Russian Academy of Sciences: Physics, year 2009, pages 343-346, volume 73, doi: 10.3103/S1062873809030198 